\title{
以全氟丁基为基础的具有高表面活性的氟表面活性剂
}

\author{
杨百勤 ${ }^{1}$ 陈 凯 ${ }^{1}$ 邢 航 $^{2}$ 肖进新 ${ }^{2, *}$ \\ (' 陕西科技大学化学与化工学院, 教育部轻化工助剂化学与技术重点实验室, 西安 710021; \\ ${ }^{2}$ 北京氟乐邦表面活性剂技术研究所, 北京 100080)
}

\begin{abstract}
摘要: 氟表面活性剂的环境和生物降解问题是最近的热点, 特别是全氟长链 $\left(\geqslant \mathrm{C}_{8}\right)$ 氟表面活性剂的应用限制乃 至禁用已成为必然趋势. 本文合成了一种以短链的全氟丁基为基础的阳离子氟表面活性剂, $N$-[3-(二甲基胺基) 丙基]全氟丁基磺酰胺盐酸盐 $\left(\mathrm{C}_{4} \mathrm{~F}_{9} \mathrm{SO}_{2} \mathrm{NH}\left(\mathrm{CH}_{2}\right)_{3} \mathrm{NH}\left(\mathrm{CH}_{3}\right)_{2}^{+} \mathrm{Cl}^{-}\right.$, 简称为 PFB-MC). 该表面活性剂适用于强酸性环境, 具有极高的表面活性, 其溶液最低表面张力 $\left(19.80 \mathrm{mN} \cdot \mathrm{m}^{-1}\right)$ 和通常的氟表面活性剂相当. 通过表面张力方法得到 了固定 $\mathrm{pH}(\mathrm{pH}=2.6-2.7)$ 情况下 PFB-MC 的表面张力 - 浓度对数 $(\gamma-\lg c)$ 曲线, 以及该 $\mathrm{pH}$ 下外加盐 $([\mathrm{NaCl}]=0.1$ $\mathrm{mol} \cdot \mathrm{L}^{-1}$ )对表面张力的影响; 并进一步研究了 $\mathrm{pH}$ 对 PFB-MC 在其临界胶束浓度 $(\mathrm{cmc}$ )前后的表面张力的影响.
\end{abstract}

关键词: 氟表面活性剂; 表面张力; 环境保护; 酸性条件; 盐效应 中图分类号: 0647.2

\section{Perfluorobutyl-Based Fluorinated Surfactant with High Surface Activity}

\author{
YANG Bai-Qin ${ }^{1} \quad$ CHEN Kai $^{1} \quad$ XING Hang ${ }^{2} \quad$ XIAO Jin-Xin ${ }^{2, *}$ \\ ('Key Laboratory of Auxiliary Chemistry and Technology for Chemical Industry, Ministry of Education, College of Chemistry and \\ Chemical Engineering, Shaanxi University of Science and Technology, Xi'an 710021, P. R. China; \\ ${ }^{2}$ Beijing FLUOBON Surfactant Institute, Beijing 100080, P. R. China)
}

\begin{abstract}
The environmentally friendliness and biodegradability of fluorinated surfactants are increasingly important and the use of long-chain $\left(\geqslant \mathrm{C}_{8}\right.$ ) perfluorinated surfactants will be evidently forbidden in future. In this work, a cationic fluorinated surfactant, $\mathrm{N}$-[3-(dimethylamino)propyl] butanesulfonamide monohydrochloride $\left(\mathrm{C}_{4} \mathrm{~F}_{9} \mathrm{SO}_{2} \mathrm{NH}\left(\mathrm{CH}_{2}\right)_{3}\right.$ $\mathrm{NH}\left(\mathrm{CH}_{3}\right)_{2}^{+} \mathrm{Cl}^{-}$, abbr. PFB-MC) based on a short fluorocarbon chain, perfluorobutyl, was synthesized. This surfactant was effective in acidic environments. It shows very high surface activity and its lowest surface tension $\left(19.80 \mathrm{mN} \cdot \mathrm{m}^{-1}\right)$ in aqueous solution can be compared with the common fluorinated surfactants. The surface tension-logarithm of concentration $(\gamma-\lg c)$ curve of PFB-MC at a constant $\mathrm{pH}(\mathrm{pH}=2.6-2.7)$ and the effect of salt addition on surface tension $\left([\mathrm{NaCl}]=0.1 \mathrm{~mol} \cdot \mathrm{L}^{-1}\right)$ at this $\mathrm{pH}$ were obtained by surface tension measurement. The effect of $\mathrm{pH}$ on the surface tension of these PFB-MC solutions below and above critical micelle concentration (cmc) was also studied.
\end{abstract}

Key Words: Fluorinated surfactant; Surface tension; Environment protection; Acidic condition; Salt effect

氟表面活性剂是迄今为止已知的表面活性剂中 表面活性最高的一种 ${ }^{[1,2]}$. 氟表面活性剂具有很多特 殊的优良性能, 如高表面活性、高热稳定性、高化学 稳定性及碳氟链既疏水又疏油 ${ }^{[3,4]}$, 从而使得氟表面 活性剂在很多领域具有普通表面活性剂无法替代的
应用价值 ${ }^{[5,6]}$. 目前常用的氟表面活性剂主要是以全 氟辛基磺酸 $\left(\mathrm{C}_{8} \mathrm{~F}_{17} \mathrm{SO}_{3} \mathrm{H}\right)$ 或全氟辛酸 $\left(\mathrm{C}_{7} \mathrm{~F}_{15} \mathrm{COOH}\right)$ 为 基础原料,但是近期的研究表明以这种全氟辛基 (酸)为基础的氟化合物具有毒性或者难于生物降 解 ${ }^{[7-9]}$. 随着环境保护意识的加强和国际上对于氟表

Received: August 25, 2009; Revised: October 9, 2009; Published on Web: October 16, 2009.

*Corresponding author. Email: xiaojinxin@pku.edu.cn; Tel: +86-10-62561871.

The project was supported by the Foundation of Shaanxi Provincial Department of Education, China (09JK364).

陕西省教育厅基金(09JK364)资助项目

C. Editorial office of Acta Physico-Chimica Sinica 
面活性剂生物降解的关注, 国际上已颁布逐步禁止 和取代使用碳链长 $\geqslant \mathrm{C}_{8}$ 的氟表面活性剂的协 议 ${ }^{[10,11]}$. 因此, 寻找全氟长链氟表面活性剂的替代品 的需求已经非常迫切. 考虑到有效降低水的表面张 力还是要依靠氟表面活性剂, 我们关注能否改变氟 表面活性剂的结构, 缩短其中含氟片断的长度, 外接 其它基团使其一方面具有氟表面活性剂的高表面 活性, 另一方面降低或避免其对环境的危害. 已有 结果 ${ }^{[12,13]}$ 表明, 当碳氟链等于 4 个碳时, 其对环境的 危害基本上可以不必考虑了. 但是普通的短链氟化 合物表面活性差, 实际应用受到很大限制. 如全氟 丁基磺酸钠 $\left(\mathrm{C}_{4} \mathrm{~F}_{9} \mathrm{SO}_{3} \mathrm{Na}\right)$ 水溶液的 $\mathrm{cmc}$ 和最低表面 张力分别为 $273 \mathrm{mmol} \cdot \mathrm{L}^{-1}$ 和 $29.72 \mathrm{mN} \cdot \mathrm{m}^{-1}$, 已不属 于传统的氟表面活性剂的范畴. 我们尝试得到含有 短碳链的全氟丁基 $\left(\mathrm{C}_{4} \mathrm{~F}_{9}\right.$ 一 $)$ 为基础的氟表面活性剂, 要求其中的碳氟片断短但表面活性足够好, 并达到 全氟长链氟表面活性剂的效果.

本文合成了一种新型的阳离子氟表面活性剂, $N$-[3-(二甲基胺基)丙基]全氟丁基磺酰胺盐酸盐 $\left(\mathrm{C}_{4} \mathrm{~F}_{9} \mathrm{SO}_{2} \mathrm{NH}\left(\mathrm{CH}_{2}\right)_{3} \mathrm{NH}\left(\mathrm{CH}_{3}\right)_{2}^{+} \mathrm{Cl}^{-}\right.$, 简称为 PFB-MC), 并用表面张力方法研究了 $\mathrm{pH}$ 以及外加盐对该表面 活性剂的表面张力的影响.

\section{1 实验部分}

\section{1 试 剂}

全氟丁基磺酰氟 $\left(\mathrm{C}_{4} \mathrm{~F}_{9} \mathrm{SO}_{2} \mathrm{~F}, 90 \%\right)$ 和 $N, N$-二甲基1,3-丙二胺 $\left(\mathrm{NH}_{2}\left(\mathrm{CH}_{2}\right)_{3} \mathrm{~N}\left(\mathrm{CH}_{3}\right)_{2}, 98 \%\right)$ 均购于美国 Alfa Aesar 公司; $\mathrm{NaCl}$ 、乙醚、三乙胺为分析纯, 均购 于北京化学试剂公司.

\section{2 表面活性剂的合成}

将 $0.12 \mathrm{~mol} \mathrm{~N}, \mathrm{~N}$-二甲基- 1,3 -丙二胺和 $0.12 \mathrm{~mol}$ 三乙胺与 $30 \mathrm{~mL}$ 乙醚混合, $20{ }^{\circ} \mathrm{C}$ 滴加 $0.1 \mathrm{~mol}$ 全氟 丁基磺酰氟, 搅拌 $1 \mathrm{~h}$ 后升温至回流, 回流 $4 \mathrm{~h}$ 后停 止反应, 静置分层, 除去上层清液, 得表面活性剂的 前体粗品 $\left(\mathrm{C}_{4} \mathrm{~F}_{9} \mathrm{SO}_{2} \mathrm{NH}\left(\mathrm{CH}_{2}\right)_{3} \mathrm{~N}\left(\mathrm{CH}_{3}\right)_{2}\right)$, 其中三乙胺是 用来吸收反应产生的 HF. 反应式如下:

\section{$\mathrm{C}_{4} \mathrm{~F}_{9} \mathrm{SO}_{2} \mathrm{~F}+\mathrm{NH}_{2}\left(\mathrm{CH}_{2}\right)_{3} \mathrm{~N}\left(\mathrm{CH}_{3}\right)_{2}+\mathrm{N}\left(\mathrm{C}_{2} \mathrm{H}_{5}\right)_{3} \underset{\text { ether }}{\overrightarrow{\mathrm{C}}}$}

$\mathrm{C}_{4} \mathrm{~F}_{9} \mathrm{SO}_{2} \mathrm{NH}\left(\mathrm{CH}_{2}\right)_{3} \mathrm{~N}\left(\mathrm{CH}_{3}\right)_{2}+\mathrm{NH}\left(\mathrm{C}_{2} \mathrm{H}_{5}\right)_{3} \mathrm{~F}$

所得产物分别用乙醚洗涤两次, 用去离子水洗 涤三次, 干燥. 产物用红外、核磁、元素分析进行了检 测, 证实了其结构. 表面张力曲线没有观察到最低 点, 证明没有高表面活性的杂质存在 ${ }^{[14]}$. 之后采用盐 酸酸化的方法制得我们所需要的有机铵盐型的阳离
子氟表面活性剂 $\left(\mathrm{C}_{4} \mathrm{~F}_{9} \mathrm{SO}_{2} \mathrm{NH}\left(\mathrm{CH}_{2}\right)_{3} \mathrm{NH}\left(\mathrm{CH}_{3}\right)_{2}^{+} \mathrm{Cl}^{-}\right.$, 简 称为 PFB-MC), 反应如下:

$$
\begin{gathered}
\mathrm{C}_{4} \mathrm{~F}_{9} \mathrm{SO}_{2} \mathrm{NH}\left(\mathrm{CH}_{2}\right)_{3} \mathrm{~N}\left(\mathrm{CH}_{3}\right)_{2}+\mathrm{HCl} \longrightarrow \\
\mathrm{C}_{4} \mathrm{~F}_{9} \mathrm{SO}_{2} \mathrm{NH}\left(\mathrm{CH}_{2}\right)_{3} \mathrm{NH}\left(\mathrm{CH}_{3}\right)_{2}^{+} \mathrm{Cl}^{-}
\end{gathered}
$$

\section{3 表面活性剂的性质测定}

采用滴体积法测定表面张力 ${ }^{[15]}$. 所有溶液均用去 离子水配制. 溶液的 $\mathrm{pH}$ 通过控制外加盐酸的量调 节. 除研究 $\mathrm{pH}$ 的影响外, 单一体系及其加盐的实验 均保证盐酸是过量的 $(\mathrm{pH}=2.6-2.7)$, 以确保表面活性 剂前体全部生成表面活性剂 PFB-MC. 表面活性剂 的临界胶束浓度 $(\mathrm{cmc})$ 和 $\mathrm{cmc}$ 处的表面张力 $\left(\gamma_{\mathrm{cmc}}\right)$ 由 表面张力-浓度对数 $(\gamma-\lg c)$ 曲线的转折点求出. 所 有实验均在 $25{ }^{\circ} \mathrm{C}$ 下测定.

\section{2 结果及讨论}

\subsection{PFB-MC 的表面活性及加盐的影响}

PFB-MC 的结构特点决定了该表面活性剂适用 于强酸性环境. 图 $1 \mathrm{a}$ 给出了 $\mathrm{pH}$ 为 2.6-2.7 时 PFB$\mathrm{MC}$ 的表面张力曲线. 从图中可以得到 PFB-MC 的 $\mathrm{cmc}=36.4 \mathrm{mmol} \cdot \mathrm{L}^{-1}$ 以及 $\gamma_{\mathrm{cmc}}=19.80 \mathrm{mN} \cdot \mathrm{m}^{-1}$. 这表明 PFB-MC 具有很高的表面活性,例如和常见的氟表 面活性剂全氟辛酸钠相比 $\left(\mathrm{C}_{7} \mathrm{~F}_{15} \mathrm{COONa}, \mathrm{cmc}=31.2\right.$ $\left.\mathrm{mmol} \cdot \mathrm{L}^{-1} ; \gamma_{\mathrm{cmc}}=24.7 \mathrm{mN} \cdot \mathrm{m}^{-1}\right)^{[16]}$, 两者 $\mathrm{cmc}$ 相差不多, PFB-MC 的 $\gamma_{\mathrm{cmc}}$ 比 $\mathrm{C}_{7} \mathrm{~F}_{15} \mathrm{COONa}$ 更低.

进一步考察了同等条件下 $(\mathrm{pH}=2.6-2.7)$ 外加盐 对表面活性剂的表面张力的影响, 如图 $1 b$ 所示. 根 据表面张力曲线可知, $\mathrm{PFB}-\mathrm{MC}$ 在 $0.1 \mathrm{~mol} \cdot \mathrm{L}^{-1} \mathrm{NaCl}$ 存在下其 $\mathrm{cmc}=14.0 \mathrm{mmol} \cdot \mathrm{L}^{-1}, \gamma_{\mathrm{cmc}}=19.74 \mathrm{mN} \cdot \mathrm{m}^{-1}$.

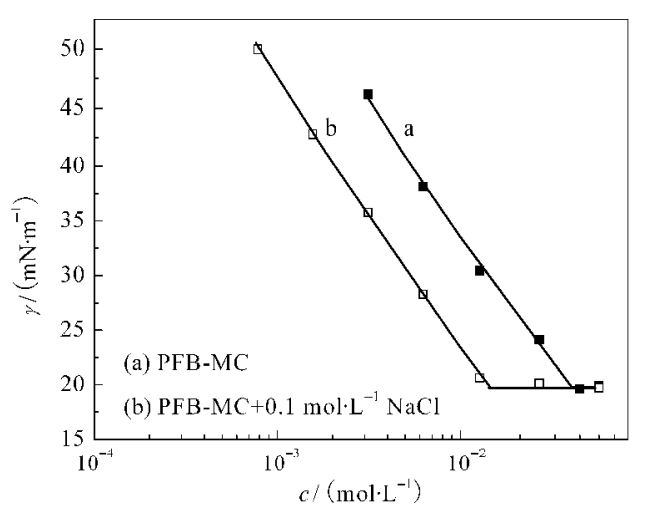

图 $1 \mathrm{pH}=2.6-2.7$ 及 $25^{\circ} \mathrm{C}$ 时 PFB-MC 加和不加 0.1 $\mathbf{m o l} \cdot \mathbf{L}^{-1} \mathbf{N a C l}$ 情况下的表面张力 $(\gamma)$ 随浓度 $(c)$ 的变化曲线

Fig.1 Surface tension $(\gamma)$ vs concentration (c) curves of PFB-MC in the absence and presence of $0.1 \mathrm{~mol} \cdot \mathrm{L}^{-1} \mathrm{NaCl}$ at $\mathrm{pH}=2.6-2.7$ and $25{ }^{\circ} \mathrm{C}$ PFB-MC: $\mathrm{C}_{4} \mathrm{~F}_{9} \mathrm{SO}_{2} \mathrm{NH}\left(\mathrm{CH}_{2}\right)_{3} \mathrm{NH}\left(\mathrm{CH}_{3}\right)_{2}^{+} \mathrm{Cl}^{-}$ 
比较加盐和不加盐的 PFB-MC 的表面张力曲线, 可 以看到无论是直线的斜率还是 $\gamma_{\mathrm{cmc}}$ 两者都很接近, 但是加盐之后 PFB-MC 的 $\mathrm{cmc}$ 比不加盐时有明显 降低.

利用 Gibbs 吸附公式 ${ }^{[14]}$ 计算溶液的饱和吸附量 $\Gamma_{\mathrm{m}}$. 由于本实验中体系始终存在过量电解质, 则有:

$$
\Gamma_{\mathrm{m}}=-\frac{1}{R T} \frac{\mathrm{d} \gamma}{\mathrm{d} \ln c}=-\frac{1}{2.303 R T} \frac{\mathrm{d} \gamma}{\mathrm{dlg} c}
$$

其中 $c$ 为表面活性剂浓度, $R$ 为摩尔气体常数, $T$ 为 热力学温度. 通过 $\gamma-\lg c$ 曲线的斜率, 代人式(1)可得 到饱和吸附量 $\Gamma_{\mathrm{m}}$, 可进一步求得吸附分子的极限面 积 $A_{\min }{ }^{[14]}$ :

$$
A_{\min }=\frac{1}{N_{0} \Gamma_{\mathrm{m}}}
$$

其中 $N_{0}$ 为 Avogadro 常数.

将 PFB-MC 加盐、不加盐溶液的 $\mathrm{cmc} 、 \gamma_{\mathrm{cmc}} 、 \Gamma_{\mathrm{m}}$ 、 $A_{\min }$ 列于表 1 . 根据表 1 数据, 加盐之后的溶液其 $\gamma_{\mathrm{cm}} 、 \Gamma_{\mathrm{m}}$ 和 $A_{\min }$ 与不加盐的溶液相比相差不大, 这可 能是由于两者溶液中已经含有过量的盐酸 $(\mathrm{pH}=$ 2.6-2.7), 并且盐酸在一定程度上起到了电解质的作 用, 这样造成了引人 $0.1 \mathrm{~mol} \cdot \mathrm{L}^{-1} \mathrm{NaCl}$ 的效果(特别 是盐的屏蔽效应)并不明显. 但是 $\mathrm{cmc}$ 在加盐之后有 明显的降低, 我们推测除了 $\mathrm{Na}^{+}$和 $\mathrm{Cl}^{-}$对 PFB-MC 头 基之间的电荷排斥起到了一定的屏蔽作用(当然, 由 于溶液中已经存在过量的 $\mathrm{H}^{+}$和 $\mathrm{Cl}^{-}$, 这种作用不是 很明显), 更重要的是 $0.1 \mathrm{~mol} \cdot \mathrm{L}^{-1} \mathrm{NaCl}$ 的引人带来 了“盐析”效果, 也就是说, 加人的 $\mathrm{NaCl}$ 夺取溶液中 的水使得表面活性剂的有效浓度增加了, 这样相当 于外加盐降低了表面活性剂的溶解性(即增强了表 面活性剂的疏水性), 所以含有 $0.1 \mathrm{~mol} \cdot \mathrm{L}^{-1} \mathrm{NaCl}$ 的 PFB-MC 溶液其 $\mathrm{cmc}$ 明显减小.

表 $1 \mathrm{pH}=2.6-2.7$ 及 $25^{\circ} \mathrm{C}$ 时 PFB-MC 加和不加 0.1 $\mathbf{m o l} \cdot \mathbf{L}^{-1} \mathrm{NaCl}$ 情况下的临界胶束浓度 $(\mathbf{c m c}) 、 \mathrm{cmc}$ 处的 表面张力 $\left(\gamma_{\mathrm{cmc}}\right)$ 、饱和吸附量 $\left(\boldsymbol{\Gamma}_{\mathrm{m}}\right)$ 及吸附分子的 极限面积 $\left(A_{\text {min }}\right)$ 对比

Table 1 Comparison of critical micelle concentration (cmc), surface tension at cmc $\left(\gamma_{\mathrm{cmc}}\right)$, maximum amount adsorbed $\left(\Gamma_{\mathrm{m}}\right)$, and minimum surface area per molecule $\left(A_{\min }\right)$ between the solutions of PFB-MC in the absence and presence of $0.1 \mathrm{~mol} \cdot \mathrm{L}^{-1} \mathrm{NaCl}$ at $\mathrm{pH}=2.6-2.7$ and $25{ }^{\circ} \mathrm{C}$

\begin{tabular}{lccccc}
\hline \multirow{2}{*}{ System } & $\begin{array}{c}\mathrm{cmc} \\
\left(\mathrm{mmol} \cdot \mathrm{L}^{-1}\right)\end{array}$ & $\frac{\gamma_{\mathrm{cmc}}}{\left(\mathrm{mN} \cdot \mathrm{m}^{-1}\right)}$ & $\frac{10^{10} \Gamma_{\mathrm{m}}}{\left(\mathrm{mol} \cdot \mathrm{cm}^{-2}\right)}$ & $\frac{A_{\min }}{\mathrm{nm}^{2}}$ \\
\hline PFB-MC & 36.4 & 19.80 & 4.30 & 0.386 \\
PFB-MC+0.1 $\mathrm{mol} \cdot \mathrm{L}^{-1} \mathrm{NaCl}$ & 14.0 & 19.74 & 4.24 & 0.392 \\
\hline
\end{tabular}

\section{$2.2 \mathrm{pH}$ 对 $\mathrm{cmc}$ 前后浓度的 PFB-MC 表面性质的 影响}

如前所述, PFB-MC 的结构特点决定了其在酸 过量的条件下才能稳定存在, 因此有必要探讨 $\mathrm{pH}$ 对其表面性质的影响, 从而确定其 $\mathrm{pH}$ 适用范围. 图 2 给出了 $\mathrm{pH}$ 对 PFB-MC 表面张力的影响. 结果表 明, 对于 $0.025 \mathrm{~mol} \cdot \mathrm{L}^{-1}$ 的 $\mathrm{PFB}-\mathrm{MC}$, 当 $\mathrm{pH}$ 较高 $(\mathrm{pH}$ 7-8)时, 表面张力很高 $\left(45 \mathrm{mN} \cdot \mathrm{m}^{-1}\right.$ 以上), 此时 PFB$\mathrm{MC}$ 主要是以前体形式 $\left(\mathrm{C}_{4} \mathrm{~F}_{9} \mathrm{SO}_{2} \mathrm{NH}\left(\mathrm{CH}_{2}\right)_{3} \mathrm{~N}\left(\mathrm{CH}_{3}\right)_{2}\right.$ 固 体)存在, 并未起到表面活性剂的作用; 随着 $\mathrm{pH}$ 的 下降 $(4.8<\mathrm{pH}<7.0)$, 表面活性剂 PFB-MC 随着盐酸 的加人其生成量明显增加, 使得表面张力有明显下 降; 而当 $\mathrm{pH}$ 进一步降低, 我们观察到 $\mathrm{pH} 3$ 附近时 溶液已经完全变清, 这时可以认为 PFB-MC 完全转 化成表面活性剂的形式, 此时表面张力下降的幅度 明显变缓, 这种下降可以理解为盐酸浓度的增加使 其屏蔽了在表面上吸附的 PFB-M+表面活性离子头 基之间的静电排斥.

此外我们还研究 $\mathrm{pH}$ 对较高浓度 $\left(0.050 \mathrm{~mol} \cdot \mathrm{L}^{-1}\right)$ PFB-MC 表面张力的影响(图 2b). 在 $\mathrm{pH}$ 较大时, 一 定 $\mathrm{pH}$ 范围内(4.5-7.0) 所观察到的现象和 0.025 $\mathrm{mol} \cdot \mathrm{L}^{-1} \mathrm{PFB}-\mathrm{MC}$ 类似, 都是随 $\mathrm{pH}$ 下降表面张力显 著下降,即表面活性剂不断生成的过程. 但是表面张 力在 $\mathrm{pH}<4$ 时基本维持不变. 对比 $\mathrm{pH}=2.6-2.7$ 时 PFB-MC 加盐及不加盐的表面张力曲线(图 1), 就不 难理解图 2 中在低 $\mathrm{pH}$ 区这两个浓度的 PFB-MC 溶 液表面张力随 $\mathrm{pH}$ 变化的差别: 根据图 $1, \mathrm{pH}=2.6-$ 2.7 时 $\mathrm{PFB}-\mathrm{MC}\left(0.025 \mathrm{~mol} \cdot \mathrm{L}^{-1}\right)$ 不加盐的表面张力还 未达到最低(此时 $\gamma=24.16 \mathrm{mN} \cdot \mathrm{m}^{-1}$ ), 而加人 $0.1 \mathrm{~mol} \cdot$

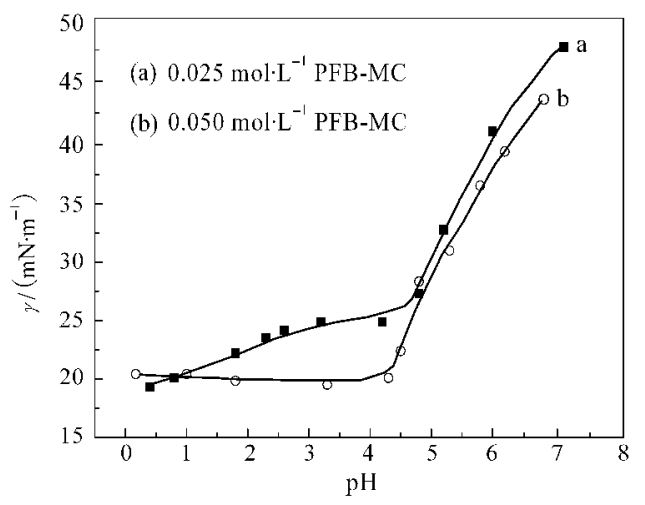

图 2 PFB-MC 在 $0.025 \mathrm{~mol} \cdot \mathrm{L}^{-1}$ (低于 $\mathrm{cmc}$ )及 $0.050 \mathrm{~mol} \cdot \mathrm{L}^{-1}$ (高于 $\mathrm{cmc}$ ) 时的表面张力随 $\mathrm{pH}$ 的变化

Fig.2 Surface tension variation with $\mathbf{p H}$ for PFB-MC at $0.025 \mathrm{~mol} \cdot \mathrm{L}^{-1}\left(\right.$ below $\mathrm{cmc}$ ) and $0.050 \mathrm{~mol} \cdot \mathrm{L}^{-1}$ (above cmc) 
$\mathrm{L}^{-1} \mathrm{NaCl}$ 之后, 观察到 $0.025 \mathrm{~mol} \cdot \mathrm{L}^{-1}$ 的 $\mathrm{PFB}-\mathrm{MC}$ 的 表面张力已达最低点 $\left(19.74 \mathrm{mN} \cdot \mathrm{m}^{-1}\right)$, 也就是说 $0.025 \mathrm{~mol} \cdot \mathrm{L}^{-1}$ 的 $\mathrm{PFB}-\mathrm{MC}$ 的表面张力在外加电解 质情况下仍有下降空间; 而 PFB-MC $\left(0.050 \mathrm{~mol} \cdot \mathrm{L}^{-1}\right)$ 始终处于表面张力最低点的状态. 所以图 2 中 0.025 $\mathrm{mol} \cdot \mathrm{L}^{-1}$ 的 $\mathrm{PFB}-\mathrm{MC}$ 在低 $\mathrm{pH}$ 区随着 $\mathrm{pH}$ 下降其表面 张力仍缓慢下降, 而 $0.050 \mathrm{~mol} \cdot \mathrm{L}^{-1}$ 的 $\mathrm{PFB}-\mathrm{MC}$ 的表 面张力则维持不变.

总之, PFB-MC 适用的最佳 $\mathrm{pH}$ 范围是 $\mathrm{pH}$ 在 3 附近以及更强酸性的环境. 在实际应用中, 常常要求 表面活性剂能在强酸环境下使用, 如工业清洗(强酸 洗涤液 $)^{[17,18]}$, 石油工业中的酸化压裂液、助排剂等 ${ }^{[19,20]}$. 本文所研究的氟表面活性剂即可满足强酸性场合下 使用的要求.

\section{3 结 论}

本文合成了一种新型的以全氟丁基为基础的、 具有高表面活性的阳离子氟表面活性剂 $\left(\mathrm{C}_{4} \mathrm{~F}_{9} \mathrm{SO}_{2} \mathrm{NH}-\right.$ $\left(\mathrm{CH}_{2}\right)_{3} \mathrm{NH}\left(\mathrm{CH}_{3}\right)_{2}^{+} \mathrm{Cl}^{-}$, 简称 PFB-MC). 该表面活性剂 适用于强酸性环境并且不易受盐度环境的干扰. 加 盐还能明显改善表面活性剂的性能、降低该表面活 性剂的 $\mathrm{cmc}$. 该氟表面活性剂由于氟碳链短, 可避免 其对环境的有害影响, 从而作为全氟辛基磺酸或全 氟辛酸类衍生氟表面活性剂的替代品. 这种物质由 于碳氟链短但是表面活性高所以符合当今氟表面活 性剂的发展方向.

\section{References}

1 Kissa, E. Fluorinated surfactants and repellents. New York: Marcel Dekker Inc., 2001

2 Griffiths, P. C.; Cheung, A. Y. F.; Jenkins, R. L.; Howe, A. M.; Pitt, A. R.; Heenan, R. K.; King, S. M. Langmuir, 2004, 20: 1161

3 Xiao, J. X.; Zhao, Z. G. Application principle of surfactants. Beijing: Chemical Industry Press, 2003: Chap. 10 [肖进新, 赵振国. 表面活性剂应用原理. 北京: 化学工业出版社, 2003: 第 10 章]
4 Abe, M. Curr. Opin. Colloid Interface Sci., 1999, 4: 354

5 Xiao, J. X.; Yao, J.; Gao, Z. Chemical Research and Application, 2002, 14: 391 [肖进新, 姚 军, 高 展. 化学研究与应用, 2002, 14: 391]

6 Dong, S. L.; Li, X.; Xu, G. Y.; Hoffmann, H. J. Phys. Chem. B, 2007, 111: 5903

7 Lofgren, C. S.; Banks, W. A.; Vander Meer, R. K.; Williams, D. F. Florida Entomologist, 1989, 72: 140

8 Chen, R. Q. Dyeing \& Finishing, 2008: 41 [陈荣圻. 印染, 2008 41]

9 Liu, C. Y.; Li, Z. J. Westleather, 2009, 31: 28 [刘春滟, 李正军. 西部皮革, 2009, 31: 28]

10 Zhang, J.; Zhang, X. Q. Textile Auxiliaries, 2009, 26: 1 [章 杰, 张晓琴. 印染助剂, 2009, 26: 1]

11 Bao, Z. M.; Ye, H. L.; Fu, X. C. Fire Science and Technology, 2008, 27: 126 [包志明, 叶宏烈, 傅学成. 消防科学与技术, 2008, 27: 126]

12 Zhang, J. Dyeing \& Finishing, 2009: 47 [章 杰. 印染, 2009: 47]

13 Australian Government/Department of Health and Ageing NICNAS. Potassium perfluorobutane sulfonate. Existing chemical hazard assessment report, 2005, Nov.

14 Zhao, G. X.; Zhu, B. Y. Principles of surfactant action. Beijing: China Light Industry Press, 2003: Chap. 3 [赵国坌, 朱㻉瑶. 表 面活性剂作用原理. 北京: 中国轻工业出版社, 2003: 第 3 章]

15 Zhu, B. Y.; Zhao, Z. G. Fundamentals of interface chemistry. Beijing: Chemical Industry Press, 1996: 26-30 [朱㻉瑶, 赵振国. 界面化学基础. 北京: 化学工业出版社, 1996: 26-30]

16 Jin, C.; Yan, P.; Wang, C.; Xiao, J. X. Acta Chimica Sinica, 2005, 63: 279 [金 辰, 严 鹏, 王晨, 肖进新. 化学学报, 2005, 63: 279]

17 Ma, H. M.; Zhu, Z. L. Cleaning World, 2005, 21: 22 [马红梅, 朱志良. 清洗世界, 2005, 21: 22]

18 Yu, C. Y. Corrosion \& Protection in Petrochemical Industry, 1997, 14: 53 [余存烨. 石油化工腐蚀与防护, 1997, 14: 53]

19 Chen, L.; Zhang, G. C. Oilfield Chemistry, 2007, 24: 672 [陈 兰, 张贵才. 油田化学, 2007, 24: 672]

20 Shi, H. X.; Zhao, L. J.; Xiang, J. P.; Gao, L. D.; Shen, H. M. Fine Chemicals, 2009, 26: 331 [史鸿金金, 赵丽君, 项菊萍, 高立定, 沈海民. 精细化工, 2009, 26: 331] 